\title{
Collisions for Schnorr's Hash Function FFT-Hash Presented at Crypto '91
}

Joan Daemen, Antoon Bosselaers, René Govaerts and Joos Vandewalle

Katholieke Universiteit Leuven, Laboratorium ESAT, Kardinaal Mercierlaan 94, B-3001 Heverlee, Belgium.

\section{Abstract}

A method is described to generate collisions for the hash function FFT-Hash that was presented by Claus Schnorr at Crypto '91. A set of colliding messages is given that was obtained by this method.

\section{Introduction}

In the Rump Session of Crypto '91 Claus Schnorr presented FFT-Hash. This is a function that hashes messages of arbitrary length into a 128 bit hash value. It consists of two rounds, where every round is the combination of a Fast Fourier Transform over $\mathrm{GF}\left(2^{16}+1\right)$ and a nonlinear recursion. It was claimed that producing a pair of messages that yield the same hashvalue is computationally infeasible. We have written a program that outputs a set of 384 bit messages that all have the same hash value for FFT-Hash. The CPU-time consumed is of the order of a few hours. An optimized version of the program is expected to take only a few minutes on a modern PC. The first collision was produced on October 3rd '91.

\section{Description of FFT-Hash}

Padding: The message is padded with a single "1" followed by a suitable number of " 0 " bits followed by the binary representation of its original length. The padded message can then be seen as the concatenation of a number of 128-bit blocks: $M_{0} \|$ $M_{1} \ldots \| M_{n-1}$. 
Algorithm for the hash function $h: H_{i}=g\left(H_{i-1} \| M_{i-1}\right)$ for $i=1, \ldots, n$. $H_{i} \in\{0,1\}^{128}$ and initial value $H_{0}=01234567$ 89ab cdef fedc ba98 76543210 (hex.). The output of $h(M)=H_{n}$.

Algorithm for the function $g$ : Let $p=2^{16}+1$. The input to $g$ is split up into 16 components $\left(e_{0}, \ldots, e_{15}\right)$ with each component $e_{i}$ consuming 16 bits. These $e_{i}$ are treated as representations of integers modulo $p$. Define the FFT-transformation $F T_{8}\left(a_{0}, \ldots, a_{7}\right)=\left(b_{0}, \ldots, b_{7}\right)$ as

$$
b_{i}=\sum_{j=0}^{7} 2^{4 i j} a_{j} \bmod p \quad \text { for } \quad i=0, \ldots, 7
$$

1. $\left(e_{0}, e_{2}, \ldots, e_{14}\right)=F T_{8}\left(e_{0}, e_{2}, \ldots, e_{14}\right)$ This step is called a FFT-step

2. $\operatorname{FOR}(i=0 ; i<16 ; i++) e_{i}=e_{i}+e_{i-1} e_{i-2}+e_{e_{i-3}}+2^{i} \bmod p$

All indices are taken modulo 16 . This step is called a recursion step.

3. Second round: repeat step 1 and 2

The output of $g$ is the 128-bit string $e_{8}\left\|e_{9} \ldots\right\| e_{15}$ where all occurrences of $p-1=2^{16}$ are substituted by 0 .

\section{Weaknesses of FFT-Hash}

1. The FFT step only affects the components with even index. For odd-indexed components no diffusion takes place.

2. The linearity of the FFT step can be used to impose certain values upon a number of output components. If for certain subsets of no more than 8 components, belonging to either the output or the input, the values are fixed, values for the remaining components can be computed such that equation 1 holds. This computation involves linear algebra alone.

3. The diffusion resulting from the recursion step can be completely eliminated by imposing 0 values to certain components. Suppose $\left(e_{0}, \ldots, e_{15}\right)$ is the 16 -tuple that has just undergone a recursion step. Suppose $e_{5}=e_{7}=0$. Suppose also that $e_{6}$ was never addressed in the indirect indexing term $e_{e_{i-3}}$, hence $e_{i-3} \neq 6 \quad(\bmod 16)$ for all $i$ at the moment they are used. Then the 12 MSB bits of $e_{6}$ only appear in the calculation for the new value of $e_{6}$. This can easily be seen because when $e_{5}=e_{7}=0$ a product term $e_{i-1} e_{i-2}$ containing $e_{6}$ must be zero. Because the 12 MSB bits of $e_{6}$ can be altered without affecting the outcome of other components when the recursion is applied, $e_{6}$ will be called isolated. This can be applied to any component. Hence isolation of a component in a recursion step requires that the 
two neighboring components are 0 and that it is not addressed in the term $e_{e_{i-3}}$ for any $i$.

\section{The Attack}

The attack is based on the fact that it is possible to isolate a component during all four steps of $g$. The colliding messages consist of 3 blocks: $M_{0}, M_{1}$ and $M_{2}$. All effort goes into the search for appropriate $M_{1}$ and $M_{2}$ values. The attack is probabilistic. A subset of messagebits are given random values thereby fixing the remaining bits through a number of imposed relations. Starting from $H_{1}=g\left(H_{0} \| M_{0}\right)$ we have:

1. Calculation of $M_{1}$. The values are chosen in a way that the second component of $M_{1}\left(=e_{9}\right)$ has a maximum probability of staying isolated throughout the calculation of $g$. Certain changes in the $12 \mathrm{MSB}$ bits of this component affect the intermediate hash value $H_{2}$ only in the second component. On the average $2^{23}$ different $H_{1}$, obtained by trying different $M_{0}$ values have to be tested. Only about $2^{11}$ of these survive a first check. For each of these remaining $M_{1}$ values $2^{15}$ trials have to be performed by varying $\phi$ (see figure).

2. Calculation of $M_{2}$. The values of $M_{2}$ are chosen in such a way that the second component of $H_{2}\left(=e_{1}\right)$ has maximum probability of being isolated and thus does not affect $H_{3}$. About $2^{22}$ different values of $\phi_{1}$ and $\phi_{2}$ have to be tried.

The figure illustrates the internal relations during the hashing process of the colliding messages. $Q$ indicates the component that is isolated throughout the whole calculation.

The first result obtained by this method was a set of 805 colliding messages (in hexadecimal notation)

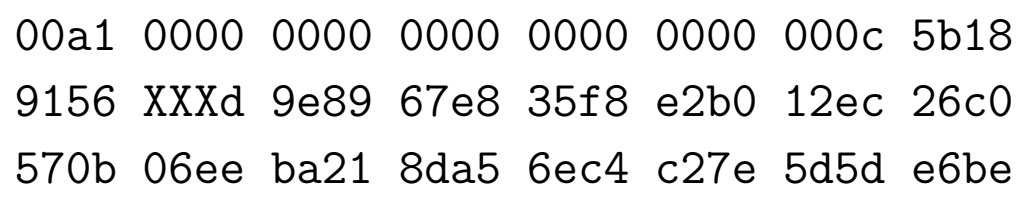

where $\mathrm{XXX}$ ranges over $1 \mathrm{~b} 5$ to $4 \mathrm{~d} 9$ that all hash to

$527 d$ c019 d8cb 1d92 162b f04c cfff $26 c 6$

\section{References}

[1] C Schnorr, FFT-Hash, An Efficient Cryptographic Hash Function, Rump Session Crypto '91. 
An arrow from $e_{i}$ to $e_{j}$ means $e_{e_{i}}=e_{j}$ or $e_{i}=j \quad(\bmod 16)$

Boxes containing a constant indicate the value that is imposed upon the component

Boxes containing a greek letter indicate variables that are isolated (denoted by $\mathbf{a}$ ) until used (as indicated in the down left corner) to impose a certain value to a component

$\mathrm{A} \star$ in the down left corner indicates that we depend upon luck (prob: $2^{-16}$ )

$\square$ indicates that the component is fixed by an FFT relation

An empty box denotes a component that is fixed by initial values and/or internal relatic
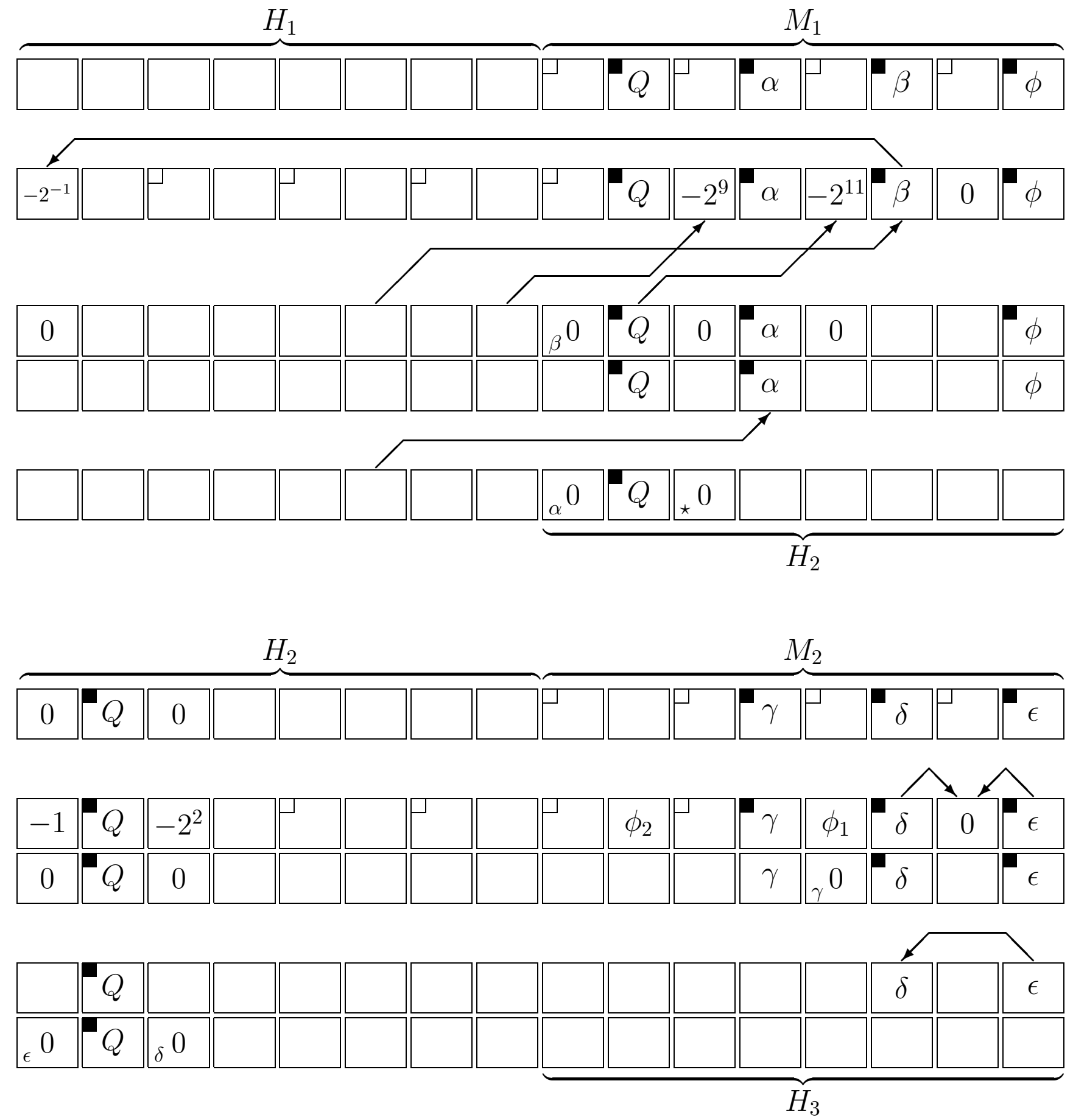

Figure 1: Schematic overview of the collisions of FFT-Hash. The state $\left(e_{0}, \ldots, e_{15}\right)$ is depicted before and after every step. 